\title{
Pinpointing the Roots of Extreme Weather Events
}

\author{
A statistical method for fingerprinting the patterns of heat waves and cold \\ spells could reveal whether climate change caused an extreme weather \\ event.
}

By Katherine Wright

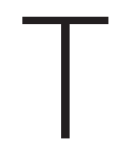

he summer of 2021 could go down as a turning point in history for its unprecedented weather. In Boise, Idaho, temperatures reached $95^{\circ} \mathrm{F}\left(35^{\circ} \mathrm{C}\right)$ and above for 24 days straight. In Lytton, Canada, which experienced a similar-length heat wave, temperatures doubled the town's summer average and topped $121^{\circ} \mathrm{F}\left(49.4^{\circ} \mathrm{C}\right)$ on June 29. In Ballywatticock, UK, record temperatures in July caused the Met Office, the body that provides the UK's weather forecasts, to issue an extreme weather warning to residents.

Climate models indicate that extreme heat waves-prolonged periods with sustained temperatures above average-will likely occur more often and with increasing severity if Earth's overall temperatures continue to rise. But teasing out whether climate change caused a specific heat wave or cold spell remains difficult. Doing so could become easier with a new method from

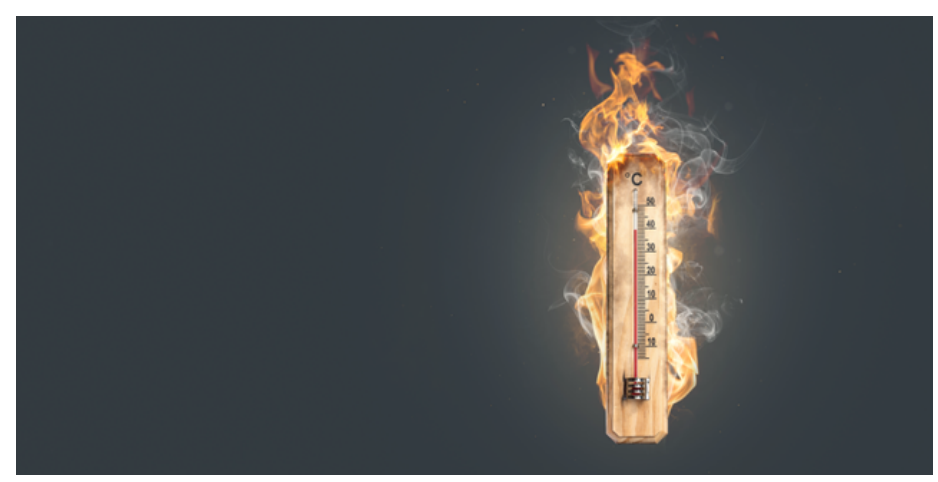

The cause of extreme weather events is difficult to pinpoint. That could change with a new statistical method.

Credit: photoschmidt/stock.adobe.com statistical physicists Valerio Lucarini of the University of Reading, UK, and Vera Gálfi of Uppsala University, Sweden. Their method allows the user to uncover what set of characteristics define an extreme weather event for a specific climate and then to use those fingerprints to determine whether a real event, such as any of the 2021 heat waves, was caused by the natural variability of the climate or by global warming.

The method combines global climate models with statistical calculations. Using a climate model and including no anthropogenic warming factors, the user first simulates Earth's climate for some time period. In the case of this study, Lucarini and Gálfi used the model whose results appeared in the most recent Intergovernmental Panel on Climate Change (IPCC) report and ran the simulations for 1000 years. The user then identifies extreme climate events that appear in the model data. For example, a month-long blast of intense heat or a deluge of rainfall that deviates from the general trend of the model would count as an extreme event. Then, the properties that define these events are characterized using large deviation theory, which is a statistical theory used in physics to evaluate the most likely situation that would constitute a rare event and how often it is likely to occur.

Finally, the patterns of the simulated extreme events are compared to real events. Similarities between the two indicate that a rare event is expected as part of Earth's natural climate variability, and differences indicate that the event is unexpected and may come from climate change.

The ability to make these inferences comes, Lucarini says, 
because of the similarity of the patterns of simulated extreme events. The analysis shows that these events all sit in the same "universality class"-a term for a collection of things that have converging properties, such as extremely high ocean waves that all have similar heights or intense summer heat waves that all have similar deviations from the mean average temperature. According to Lucarini, that universality gives the methodology predictive power and is important for another reason. "It tells us that we can have a predictive climate science," he says.

The predictive power of the model also sets it apart from others that aim to pinpoint the cause of a specific extreme climate event. Lucarini says that other methods are largely empirical, meaning that the tools can indicate the likelihood that a past extreme event was caused by climate change but cannot predict the probable occurrence of future such events. "Our methodology allows us to do that," he says.

Making such predictions is key to society's ability to make informed decisions on everything from whether individuals need to purchase fire insurance to what kinds of food a community should grow, Lucarini says. For example, food prices can change when the average outdoor temperature slightly exceeds or falls below the seasonal average because that temperature fluctuation influences crop yields. But a normal year's price fluctuations are tiny compared to years when a catastrophic heat wave wipes out crops in a region. Crop collapse is "a totally different level of problem for livelihoods and for the global market, so knowing the risk of it happening is important," Lucarini says.
The team tested the model for two extreme temperature events from 2010: a month-long heat wave in Russia, where temperatures soared to $20^{\circ} \mathrm{F}\left(11^{\circ} \mathrm{C}\right)$ above average, and a cold spell in Mongolia, which brought heavy snow and temperatures of $-58^{\circ} \mathrm{F}\left(-50^{\circ} \mathrm{C}\right)$. Similar events have been previously recorded in these regions, Lucarini says, so he and his colleagues wanted to see what their model predicted about their probable origin. The team found that both events were expected from the natural variability of the climate. However, climate change will likely make future heat waves in these regions more extreme. Next, the team intends to analyze this year's Canadian heat wave. "Our model shows that the western region of Canada can have very strong heat waves [without climate change]," Lucarini says. "But this [current] one seems out of proportion." Cecile Penland and Joseph Barsugli of the National Oceanic and Atmospheric Administration's Physical Sciences Laboratory, Colorado, write in an email that "It is difficult to identify any extreme climate event as unambiguously due to climate change. Therefore, the more objective, independent techniques used to query the problem, the better. Large deviation (LD) theory is one such technique and is seldom used by climate scientists. We hope that this [new result] will increase the visibility of LD among climate scientists."

Katherine Wright is the Deputy Editor of Physics. 